\title{
Focal Ligamentum Flavum Hypertrophy with Ochronotic Deposits: An Unusual Cause for Neurogenic Claudication in Alkaptonuria
}

\author{
Rajesh Reddy, Mudumba Vijayasaradhi, Debabrat Biswal \\ Department of Neurosurgery, Nizam's Institute of Medical Sciences, Hyderabad, India
}

Neurogenic claudication resulting from focal hypertrophy of the ligamentum flavum in the lumbar spine due to ochronotic deposits has not been reported till date. The authors discuss one such case highlighting the pathogenesis, histological and radiological features. Salient features of management are also emphasized upon.

Key Words: Spinal stenosis, Ligamentum flavum, Alkaptonuria

\section{Introduction}

Alkaptonuria is a rare, autosomal recessive disorder in which accumulation of the homogentisic acid takes place, and gets deposited in the connective tissues resulting in pigmentation of connective tissue (ochronosis). Spinal involvement in alkaptonuria is common, lumbar spine being the most common site in the form of early degenerative changes with disc space involvement [1]. Lumbar spinal stenosis in an alkaptonuria patient due to 'ligamentum flavum hypertrophy associated with ochronotic deposits' has not been reported till date. One such case is discussed along with the pathogenetic mechanisms, radiological features and the salient features of management.

\section{Case Report}

A fifty year old gentleman, a known case of ochronosis diagnosed 20 years ago, presented with history of low back ache for 10 years with progressively worsening neurogenic claudication of three years duration. No history of bowel and bladder disturbances. No past history of surgery or trauma to the spine. Upon physical examination, he had pigmentation of the sclera (Fig. 1A) and the nodular deposits over the pinna (Fig. 1B). There was darkening of the urine noted on exposure to the atmosphere (Fig. 1C). Physical examination of the spine revealed loss of lumbar lordosis. Weakness of extensor hallucis longus of both lower limbs was noted with reduced sensations in bilateral L4 and L5 dermatomes. Deep tendon reflexes were sluggish in lower limbs and plantar response was flexor. The claudication distance was around 10 metres.

A plain radiograph of the spine revealed loss of lumbar lordosis, osteoporosis, osteophyte formation, calcification of the intervertebral disc with vacuum phenomenon (Fig. 2A). Magnetic resonance imaging (MRI) of the lumbar spine revealed degenerative changes in the disc space with focal lumbar canal stenosis at the L4-5 level due to hypertrophied ligamentum flavum (Fig. 2B).

The patient underwent decompressive laminectomy for management of focal canal stenosis at L4-5 level. Perioperatively, ligamentum flavum at L4-5 level was hypertrophied, studded with blackish pigment and calcified, causing compression over the thecal sac (Fig. 3B). Pigmentation was also noted in the connective tissues (lumbosacral fascia, tendinous part of the paraspinal muscles) as well as the lamina and spinous process (Fig. 3A). Histopathological examination revealed pigment deposition in between col-

Received Feb 1, 2011; Revised Mar 20, 2011; Accepted Apr 14, 2011

Corresponding author: Rajesh Reddy, MS

Department of Neurosurgery, Nizam's Institute of Medical Sciences,

F-16/A, Madhura Nagar, Hyderabad 500038, Andhra Pradesh, India

Tel: +91-40-23732434, Fax: +91-40-23746833, E-mail: rajesh_jipmer@rediffmail.com

Copyright (C) 2012 by Korean Society of Spine Surgery which permits unrestricted non-commercial use, distribution, and reproduction in any medium, provided the original work is properly cited. Asian Spine Journal • pISSN 1976-1902 eISSN 1976-7846 
lagen bundles. The patient improved symptomatically after surgery with improvement in the claudication distance to 500 metres at 3 months follow-up and continues to remain symptomatically better at 1 year follow-up.

\section{Discussion}

Alkaptonuria is the result of loss of function, missense mutations of the gene on chromosome $3 q$ that codes for homogentisate 1, 2 dioxygenase (HGA). Scribonius first described the urinary manifestations of the disease in 1584, and Boedeker in 1858 recognized the presence in urine of a reducing substance (alkapton) with an affinity for oxygen in an alkaline medium. Virchow noted the ochre (yellow)coloured pigmentation in histological sections of postmortem tissues that lead to the term "ochronosis" [2].

The pathogenesis of the spinal manifestations in the lumbar region can be explained as follows. Raised blood levels of homogentisic acid lead to deposition of pigmented benzoquinone polymeric oxidation products of homogentisic in chondrocytes, type 2 collagen fibres of the ligaments and elastic cartilages [3]. The axial loading of the body weight is maximum at the lower lumbar spine, because of which it undergoes early and accelerated age related degenerative changes. This disrupts the integrity of the spinal stabiliz-

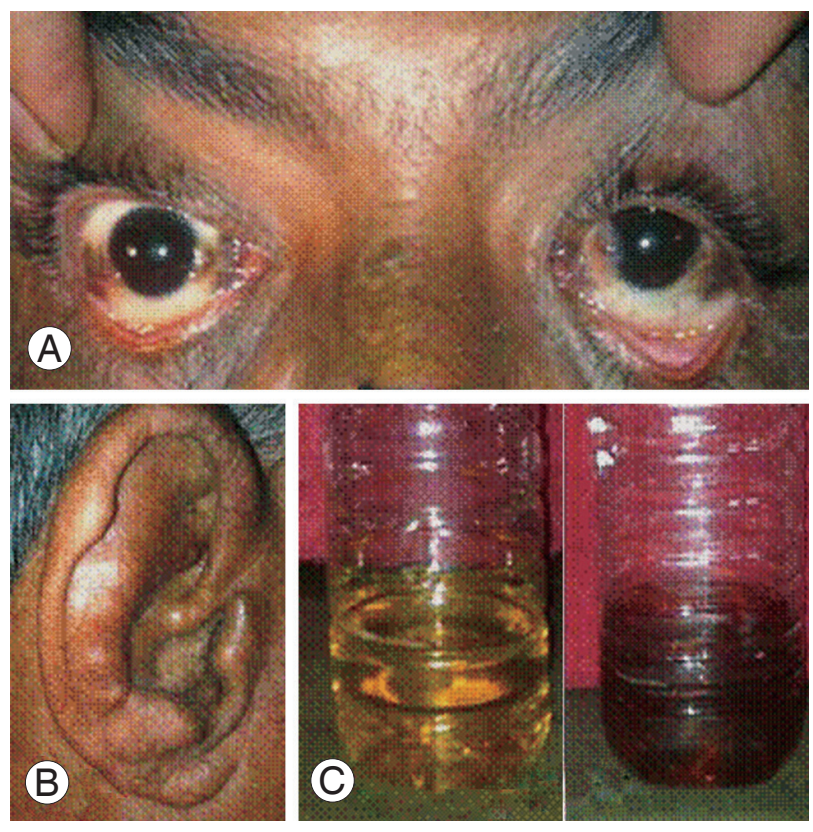

Fig. 1. (A) Brownish black pigmentation of the sclera noted in both eyes on either side of the cornea. (B) Nodules over the ear cartilage due to pigment deposition. (C) Darkening of urine on exposure to the atmospheric air as a result of oxidation of homogentisic acid to benzoquinone acetate. ing systems - passive (disc, ligament, bone, and passive muscle), active (tendons and active muscle) and neural (the nervous system and neural components within the passive and active structures), causing a transfer in unfavorable loads onto other spinal structures [4]. The presence of a calcified disc and stiff spine anteriorly causes transfer of mechanical forces to the posterior ligamentous structures of the spine, resulting in increased expression of tissue inhibitors of matrix metallaprotinase-2 in ligamentum flavum fibroblasts with resultant fibrosis and hypertrophy of the ligamentum flavum [5]. The ochronotic deposits accelerate further calcium deposition in the ligaments enhancing their stiffness. Vacuum disc phenomenon presumably represents areas of severe degeneration within the intervertebral disc [6]. Our patient had calcificied intervertebral discs at L2/3 and L3/4 which would have resulted in transfer of axial load at these immobile levels to the posterior elements at L4/5 level, which is the mobile segment in the immediate vicinity below.

A preoperative computed tomographic (CT) examination helps in identifying calcified disc protrusion (elliptical wafer appearance), ossified ligamentum flavum, osteophytes or degenerated facets which would necessitate the use of a high speed drill intraoperatively to remove the compressing element. The striking feature on MRI is multiple level disc prolapse, most common in the lumbar spine, with uniformly prominent low signal on T2WI in all discs suggesting disc degeneration and "Universal Calcification". The other findings include Schmorl nodules in the cartilage endplates, bone cysts $[7,8]$.

Irrespective of the pathogenesis, surgical decompression of the offending element causing spinal stenosis (protruded disc or hypertrophied ligamentum flavum) remains the treatment modality. Protein restriction and treatment with ascorbic acid may reduce plasma HGA levels, but treatment with nitisinone, which blocks HGA production and reduces HGA levels, is currently considered to be the best hope for treatment of these patients.

Disc prolapse in lumbar and dorsal spine of ochronotic patients has been reported previously [1,8-10]. In all the case reports of lumbar and dorsal disc prolapse in ochronotic patients published in literature, there is no mention of ligamentum flavum hypertrophy associated with ochronotic deposits. Hence, this unusual spinal manifestation of hypertrophied ligamentum flavum with ochronotic depostis should be considered in the differential diagnosis of alkaptonuria patients presenting with neurogenic claudication. 

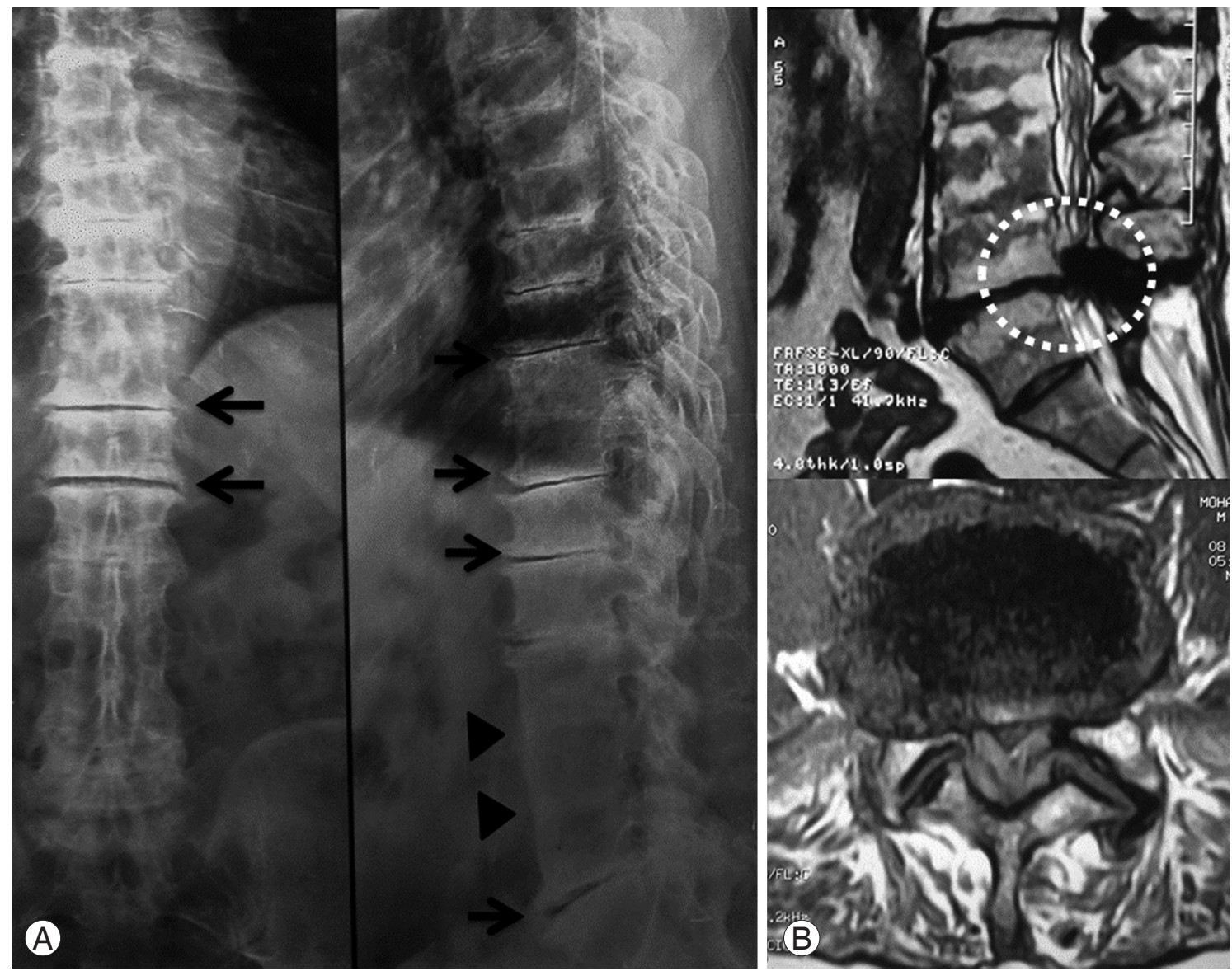

Fig. 2. (A) Plain radiograph of the dorsolumbar spine showing loss of lumbar lordosis, osteoporosis, osteophyte formation, calcification of the intervertebral disc (arrowheads) with vacuum phenomenon (arrows). (B) MRI of the lumbar spine showing degenerative changes in the vertebral end plate and focal stenosis at L4-5 level due to hypertrophy of the ligamentum flavum which appears hypointense on T2WI (encircled), T1WI and Fat suppression images (not depicted in picture).
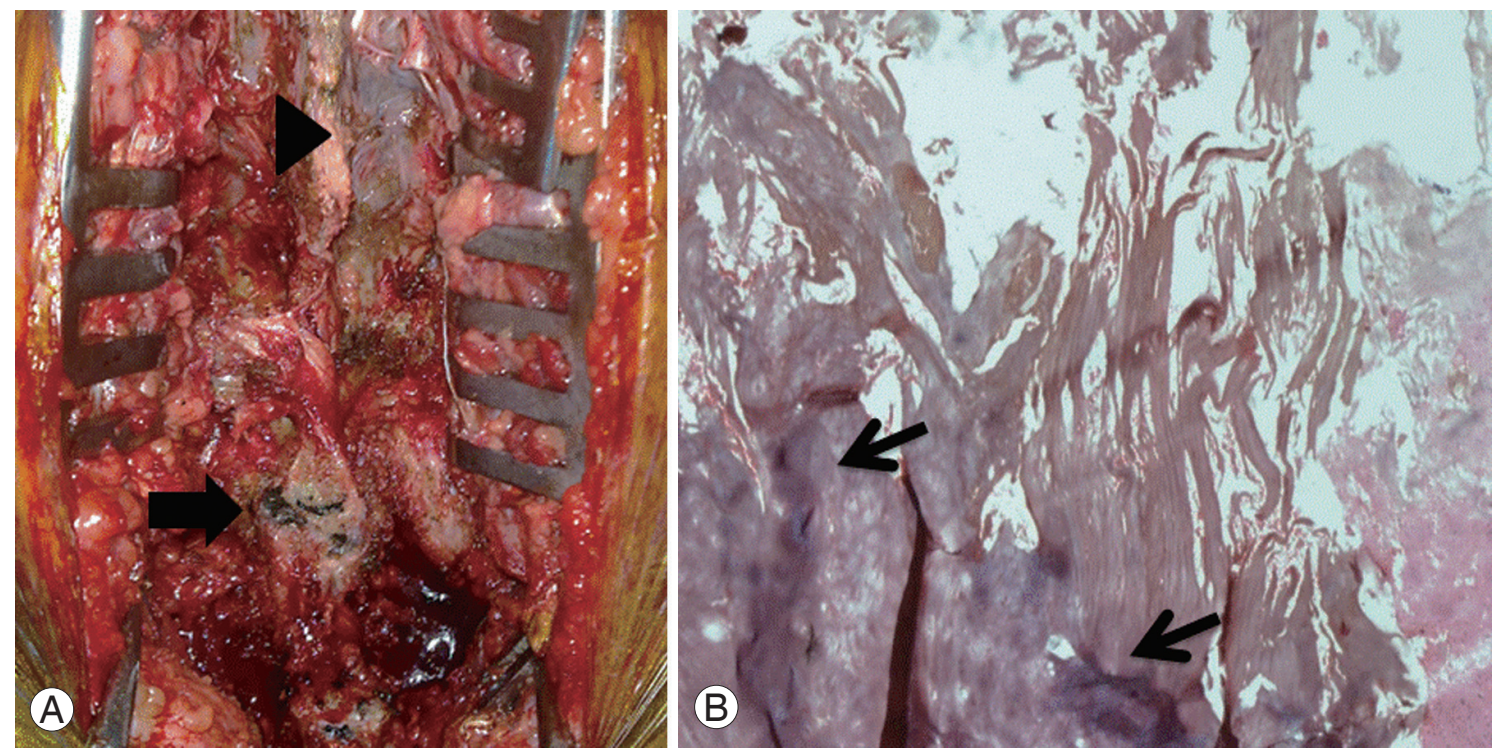

Fig. 3. (A) Intraoperative photograph showing discoloration of the fascia over the paraspinal muscles (arrowhead) and the level of cord compression by ossified, pigmented ligamentum flavum (arrow). (B) Photomicrograph $(\mathrm{H} \& \mathrm{E}, \times 10)$ demonstrating pigment deposition between collagen bundles of ligamentum flavum (arrows). 
Such patients should be evaluated with both MRI and CT scan of the involved segment for reasons discussed earlier.

\section{REFERENCES}

1. Kalevski SK, Haritonov DG, Peev NA. Alcaptonuria with lumbar disc prolapse: case study and review of the literature. Spine J 2007;7:495-8.

2. O’Brien WM, La Du BN, Bunim JJ. Biochemical, pathologic and clinical aspects of alkaptonuria, ochronosis and ochronotic arthropathy: review of world literature (1584-1962). Am J Med 1963;34:813-38.

3. Helliwell TR, Gallagher JA, Ranganath L. Alkaptonuria: a review of surgical and autopsy pathology. Histopathology 2008;53:503-12.

4. Panjabi MM. The stabilizing system of the spine. Part I. Function, dysfunction, adaptation, and enhancement. J Spinal Disord 1992;5:383-9.

5. Park JB, Lee JK, Park SJ, Riew KD. Hypertrophy of ligamentum flavum in lumbar spinal stenosis associated with increased proteinase inhibitor concentration. $\mathrm{J}$ Bone Joint Surg Am 2005;87:2750-7.

6. Deeb Z, Frayha RA. Multiple vacuum discs, and early sign of ochronosis. Radiologic findings in two brothers. J Rheumatol 1976;3:82-7.

7. Choudhury R, Rajamani SS, Rajashekhar V. A case of Ochronosis: MRI of lumbar spine. Neuroradiology 2000;42:902-7.

8. Gurkanlar D, Daneyemez M, Solmaz I, Temiz C. Ochronosis and lumbar disc herniation. Acta Neurochir (Wien) 2006;148:891-4.

9. Emel E, Karagöz F, Aydín IH, Hacísalihoğlu S, Seyithanoğlu MH. Alkaptonuria with lumbar disc herniation: a report of two cases. Spine (Phila Pa 1976) 2000;25:2141-4.

10. Akeda K, Kasai Y, Kawakita E, et al. Thoracic myelopathy with alkaptonuria. Spine (Phila Pa 1976) 2008;33:E62-5. 